\title{
A Multi-wavelength Analysis of Dust and Gas in the SR 24S Transition Disk
}

\author{
P. Pinilla ${ }^{1}$, L. M. Pérez ${ }^{2}$, S. Andrews ${ }^{3}$, N. van der Marel ${ }^{4}$, E. F. van Dishoeck ${ }^{5,6}$, S. Ataiee ${ }^{7}$, M. Benisty ${ }^{8}$, \\ T. Birnstiel ${ }^{9}$, A. Juhász ${ }^{10}$, A. Natta ${ }^{11,12}$, L. Ricci ${ }^{3,13}$, and L. Testi ${ }^{12,14}$ \\ ${ }^{1}$ Department of Astronomy/Steward Observatory, The University of Arizona, 933 North Cherry Avenue, Tucson, AZ 85721, USA; pinilla@email.arizona.edu \\ ${ }^{2}$ Max-Planck-Institut für Radioastronomie, Auf dem Hügel 69, D-53121 Bonn, Germany \\ ${ }^{3}$ Harvard-Smithsonian Center for Astrophysics, 60 Garden Street, Cambridge, MA 02138, USA \\ ${ }^{4}$ Institute for Astronomy, University of Hawaii at Manoa, Honolulu, HI, USA \\ ${ }^{5}$ Leiden Observatory, Leiden University, P.O. Box 9513, 2300RA Leiden, The Netherlands \\ ${ }_{7}^{6}$ Max-Plank-Institut für Extraterrestrische Physik, Giessenbachstraße 1, D-85748 Garching, Germany \\ ${ }^{7}$ Center for Space and Habitability, Physikalisches Institut, Universitaet Bern, 3012 Bern, Switzerland \\ ${ }^{8}$ Univ. Grenoble Alpes, CNRS, IPAG, F-38000 Grenoble, France \\ ${ }^{9}$ University Observatory, Faculty of Physics, Ludwig-Maximilians-Universität München, Scheinerstr. 1, 81679 Munich, Germany \\ ${ }^{10}$ Institute of Astronomy, Madingley Road, Cambridge CB3 OHA, UK \\ ${ }^{11}$ Dublin Institute for Advanced Studies, School of Cosmic Physics, 31 Fitzwilliam Place, Dublin 2, Ireland \\ ${ }^{12}$ INAF-Arcetri, Largo E. Fermi 5, I-50125 Firenze, Italy \\ ${ }^{13}$ Department of Physics and Astronomy, Rice University, 6100 Main Street, 77005 Houston, TX, USA \\ ${ }^{14}$ European Southern Observatory, Karl-Schwarzschild-Str. 2, D-85748 Garching, Germany \\ Received 2016 December 23; revised 2017 March 17; accepted 2017 March 24; published 2017 April 20
}

\begin{abstract}
We present new Atacama Large Millimeter/sub-millimeter Array (ALMA) $1.3 \mathrm{~mm}$ continuum observations of the SR 24S transition disk with an angular resolution $\lesssim 0$ '.18 (12 au radius). We perform a multi-wavelength investigation by combining new data with previous ALMA data at $0.45 \mathrm{~mm}$. The visibilities and images of the continuum emission at the two wavelengths are well characterized by a ring-like emission. Visibility modeling finds that the ring-like emission is narrower at longer wavelengths, in good agreement with models of dust-trapping in pressure bumps, although there are complex residuals that suggest potentially asymmetric structures. The $0.45 \mathrm{~mm}$ emission has a shallower profile inside the central cavity than the $1.3 \mathrm{~mm}$ emission. In addition, we find that the ${ }^{13} \mathrm{CO}$ and $\mathrm{C}^{18} \mathrm{O}(J=2-1)$ emission peaks at the center of the continuum cavity. We do not detect either continuum or gas emission from the northern companion to this system (SR 24N), which is itself a binary system. The upper limit for the dust disk mass of SR $24 \mathrm{~N}$ is $\lesssim 0.12 M_{\oplus}$, which gives a disk mass ratio in dust between the two components of $M_{\text {dust,SR 24S }} / M_{\text {dust,SR 24N }} \gtrsim 840$. The current ALMA observations may imply that either planets have already formed in the SR $24 \mathrm{~N}$ disk or that dust growth to millimeter sizes is inhibited there and that only warm gas, as seen by rovibrational $\mathrm{CO}$ emission inside the truncation radii of the binary, is present.
\end{abstract}

Key words: accretion, accretion disks - circumstellar matter - planet and satellites: formation protoplanetary disks

\section{Introduction}

Recent multi-wavelength observations of protoplanetary disks revealed astonishing structures, such as concentric dust rings, spiral arms, and asymmetries (e.g., van der Marel et al. 2013; ALMA Partnership et al. 2015; Casassus et al. 2015; Andrews et al. 2016; de Boer et al. 2016; Ginski et al. 2016; Pérez et al. 2016; Stolker et al. 2016; Fedele et al. 2017). These observations suggest that significant evolution has taken place and that probably planets have already imprinted their existence in the parental disks. Transition disks (TD) have been of particular interest due to their inner cavities, which were first identified by the lack of infrared emission (Strom et al. 1989). Different mechanisms have been proposed for the origin of TD cavities, including photoevaporation, magnetorotational instabilities (MRI), and planet-disk interaction (e.g., Regály et al. 2012; Zhu et al. 2012; Alexander et al. 2014; Flock et al. 2015; Dipierro et al. 2016; Pinilla et al. 2016b). To understand whether one or several mechanisms dominate the evolution, it is crucial to spatially resolve protoplanetary disks at different wavelengths because each physical process (or the combination of several of them) leads to different structures for the small/large dust and for the gas (e.g., Rosotti et al. 2013).

For instance, when a planet opens a gap in a gaseous disk, at the outer edge of the gap the gas density increases and the pressure has a local maximum where dust particles stop their fast radial drift and accumulate (e.g., Whipple 1972, p. 211; Pinilla et al. 2012b). This process can lead to a spatial difference for the distribution of small (micron-sized) particles, which are well-coupled to the gas, and large (millimeter-sized) particles. As a consequence, smaller and less depleted cavities or gaps are expected in the gas and small grains than in the large millimeter dust particles. In this scenario, the possibility of observing rings and cavities in the dust at different wavelengths strongly depends on the disk viscosity (de Juan Ovelar et al. 2016). Similarly, the formation of a broad and robust pressure bump that can rise from MRI processes, such as dead zones together with MHD winds, can lead to comparable structures in the gas and dust, as in the case of planet-disk interaction (Pinilla et al. 2016b).

Recent ALMA gas and dust observations of TDs show that, in most cases, there is gas inside the millimeter dust cavity. The gas usually also features a cavity, but with a lower depletion factor than the millimeter emission (e.g., Bruderer et al. 2014; Pérez et al. 2015; van der Marel et al. 2015, 2016; Canovas et al. 2016). In this paper, we report ALMA observations at 1.3 $\mathrm{mm}$ of the transition disk around SR 24S of the dust-continuum emission and the molecular lines ${ }^{13} \mathrm{CO}(J=2-1)$ and $\mathrm{C}^{18} \mathrm{O}$ 
$(J=2-1)$. For the analysis, we combine these new data with previous ALMA data at $0.45 \mathrm{~mm}$.

SR 24 is a hierarchical triple system located in the L1688 dark cloud in the Ophiuchus star formation region. L1688 extends over a range of distances, likely between 120 and 145 pc (Loinard et al. 2008; Mamajek 2008). In this paper, we adopt a value of $137 \mathrm{pc}$. Each of the components of SR 24 was identified as a T-Tauri star (Wilking et al. 1989; Ghez et al. 1993), with infrared excess (Greene et al. 1994). The separation between the two main components of SR 24 is 5!" 2 at a position angle (PA) of $348^{\circ}$ (Reipurth \& Zinnecker 1993). The northern component, SR $24 \mathrm{~N}$, is itself a binary system with a separation of $0 . ! 2$ at a PA of $87^{\circ}$ (Simon et al. 1995). The primary component SR $24 \mathrm{~S}$ is a $\mathrm{K} 2$ star and its mass is $>1.4 M_{\odot}$, while the stars in SR 24N are a K4-M4 star with a mass of $0.61_{-0.27}^{+0.6} M_{\odot}$ and a K7-M5 star with a mass of $0.34_{-0.18}^{+0.46} M_{\odot}$ (Correia et al. 2006).

SR 24S and SR 24N show similar infrared emission, indicating warm dust in the inner part of both disks (Stanke \& Zinnecker 2000; Bontemps et al. 2001). Brown et al. (2013) reported rovibrational $\mathrm{CO}$ emission at $4.7 \mu \mathrm{m}$ tracing warm gas in the inner parts of both disks (SR 24S and SR 24N). In addition, both circumprimary (SR 24S) and circumsecondary (SR 24N) disks were resolved in the infrared image obtained with the adaptive optics coronagraph CIAO (in the Subaru telescope). These observations show that the primary disk is more extended than the secondary and the disks seem to be extended enough to fill the effective Roche radius of the system (Mayama et al. 2010). SR 24S and SR 24N are highly accreting; the accretion rates obtained from the hydrogen recombination lines are for SR 24N $10^{-6.9} M_{\odot}$ year $^{-1}$ and for SR 24S $10^{-7.15} M_{\odot}$ year $^{-1}$ (Natta et al. 2006).

However, only the southern component, SR 24S, has been detected in the dust continuum (Nuernberger et al. 1998). Andrews \& Williams (2005) reported SMA observations of SR 24 at $225 \mathrm{GHz}(1.3 \mathrm{~mm})$ continuum and ${ }^{12} \mathrm{CO}(J=2-1)$ line emission. Stronger CO emission was seen around SR 24N than around SR 24S. Later SMA observations of SR 24S at $0.88 \mathrm{~mm}$ detected a dust cavity of $\sim 32$ au radius (Andrews et al. 2010), revealing that SR $24 \mathrm{~S}$ is a transition disk. van der Marel et al. (2015) reported ALMA Cycle 0 observations of the continuum and ${ }^{12} \mathrm{CO}(J=6-5)$ at $0.45 \mathrm{~mm}$. The $\mathrm{CO}$ observations of SR 24S were affected by extended emission and foreground absorption from the dark cloud in Ophiuchus, and it was not possible to infer the amount of gas and its distribution inside the dust cavity. The dust cavity size was inferred to be $25 \mathrm{au}$ from the fitting of the ALMA Cycle 0 continuum observations.

This paper is organized as follows. In Sections 2 and 3, we summarize the details of the ALMA observations, data reduction, and imaging. Section 4 presents the analysis of the data, particularly the continuum emission and the comparison with previous ALMA observations at $0.45 \mathrm{~mm}$. The discussion and main conclusions are in Sections 5 and 6, respectively.

\section{Observations}

SR 24 was observed with ALMA in Band 6 during Cycle 2 on September 26th, 2015 (\#2013.1.00091.S). For these observations 34 antennas were used and the longest baseline was $2269.9 \mathrm{~m}$. The source was observed in four spectral windows, each with a bandwidth of $1875.0 \mathrm{MHz}$. Two of them were chosen with a smoothed resolution of $976.563 \mathrm{kHz}$, centered at $219.56035 \mathrm{GHz}$ for the $\mathrm{C}^{18} \mathrm{O}(J=2-1)$ transition and $220.39868 \mathrm{GHz}$ for the ${ }^{13} \mathrm{CO}(J=2-1)$ transition, for a channel width of $\sim 1.35 \mathrm{~km} \mathrm{~s}^{-1}$ for the two lines. The other spectral windows were configured to obtain the continuum emission centered at $235 \mathrm{GHz}(\sim 1.3 \mathrm{~mm})$. The quasar QSO J1517-2422 was observed for bandpass calibration, while the quasars QSO J1617-2537 and QSO J1627-2426 were observed for phase calibration. The asteroid Pallas was observed for the flux calibration. The total observation time was $49.31 \mathrm{~min}$, with a total time on source of $22.67 \mathrm{~min}$. The data were calibrated using the Common Astronomy Software Package (CASA), version 4.4. For the reduction, there was one antenna flagged due to strange/elevated $T_{\text {sys }}$.

For imaging, the data were correctly centered by two independent procedures. First, to find the center of the image, position angle (PA), and disk inclination ( $i$ ), a simple Gaussian or disk model was used to fit the data (using uvmodelfit in CASA), either using only short baselines $(\lesssim 200 k \lambda)$ or all the uv coverage. The obtained PA and inclination for both models of the disk are $27^{\circ} .8 \pm 1.3$ and $49^{\circ} .8 \pm 2.4$, respectively. We applied the same procedure for fitting the $0.45 \mathrm{~mm}$ data with uvmodelfit, finding $P A=26^{\circ} .9 \pm 11^{\circ} .6$ and $i=47^{\circ} .2 \pm 3{ }^{\circ} .1$, in agreement with the values found by van der Marel et al. (2015). Second, since the image does not have significant asymmetries, the center is also checked by minimizing the rms scatter of the imaginary part of the visibilities around zero. Both methods give very similar centers, and $\alpha_{2000}=16: 26: 58.5$, $\delta_{2000}=-24: 45: 37.2$ were used to correct the phase center and obtain the visibilities using fixvis. The same procedure was used for previous ALMA observations at $0.45 \mathrm{~mm}$. Nevertheless, the center, PA and $i$ are again taken as free parameters when the analysis is done in the visibility domain (Section 4). Different studies show that visibility modeling has some advantages over imaging analysis, since it can identify unresolved structures and better constrain the disk morphology, without being limited by deconvolution issues that may arise during imaging (e.g., Pérez et al. 2014; Walsh et al. 2016; White et al. 2016; Zhang et al. 2016).

Continuum and line imaging were performed using the clean algorithm. We used natural weighting and Briggs weighting (robust $=0.5$ ) to find the best compromise between resolution and sensitivity. For the continuum, with Briggs weighting, we achieved a rms of $63 \mu \mathrm{Jy} \mathrm{beam}^{-1}$ with a beam size of 0 ". $18 \times 0$ ". 12 . The continuum was subtracted from linecontaining channels using uvcontsub. Since the ${ }^{13} \mathrm{CO}$ and the $\mathrm{C}^{18} \mathrm{O}$ are weak detections, we performed natural weighting for a final rms of around $1.4 \mathrm{mJy} \mathrm{beam}^{-1}$ per $1.35 \mathrm{~km} \mathrm{~s}^{-1}$ channel for both lines, and the final beam size in this case is 0 ". $21 \times 0$ ". 16 .

\section{Results}

\subsection{Continuum Emission}

Figure 1 shows the resulting $1.3 \mathrm{~mm}$ image of the SR 24 system after the cleaning process (and after primary beam correction). In the left panel, the contour lines correspond to $3 \sigma_{N}$, where $\sigma_{N}=75 \mu \mathrm{Jy}_{\text {beam }^{-1}}$ is the rms measured at the location of the north component (around 16:26:58.4424:45:31.9, Cutri et al. 2003). Taking a circular area with a radius of $1^{\prime \prime}$ and centered at the position of SR $24 \mathrm{~N}$, the total flux is $\sim 3.0 \sigma_{N}$. Assuming optically thin emission, the dust 


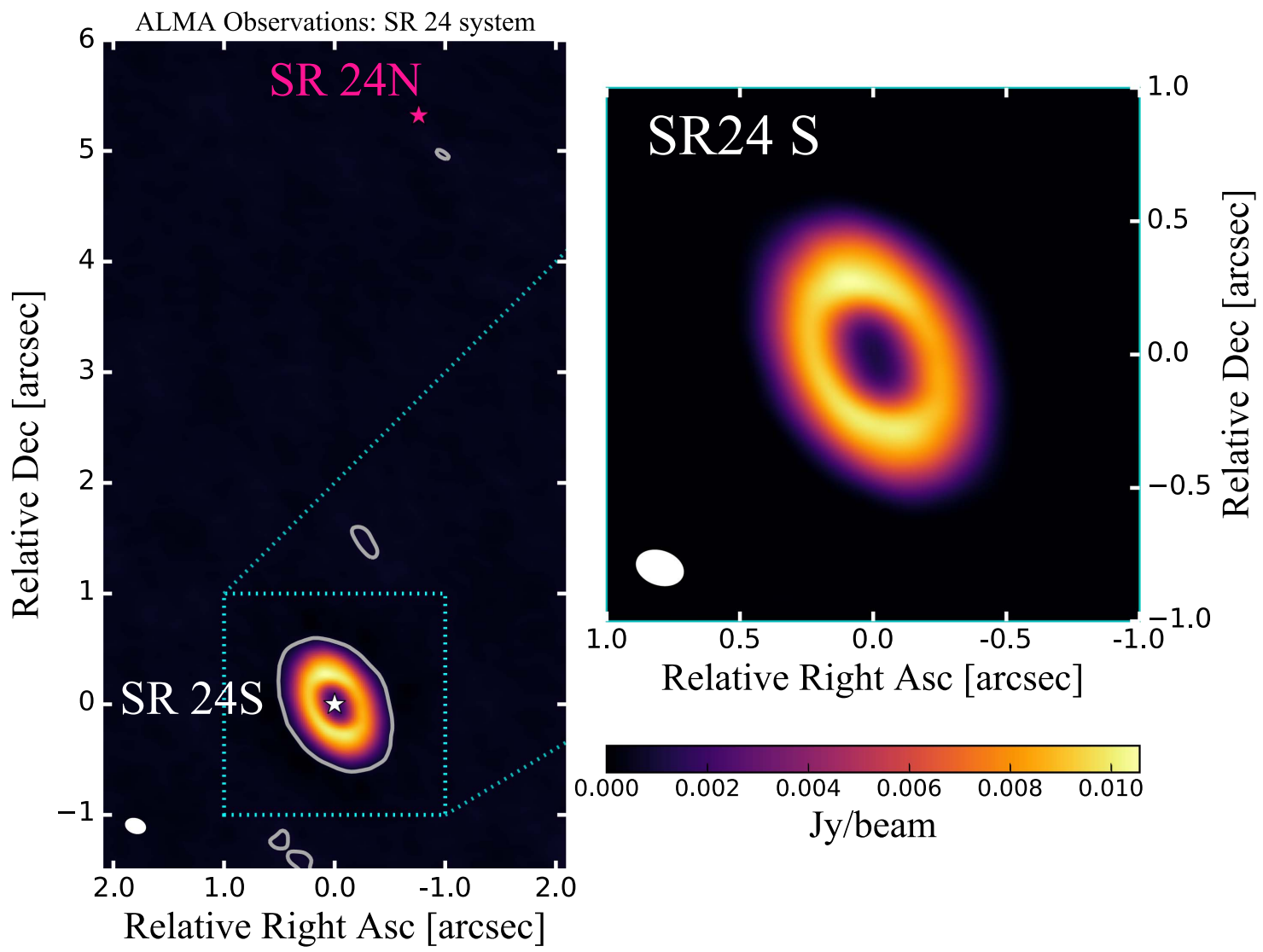

Figure 1. ALMA dust-continuum observations of the SR 24 system at $1.3 \mathrm{~mm}$, with a resolution of 0 !" $18 \times 0$ !" 12 . The contour lines in the left panel correspond to $3 \sigma_{N}$, where $\sigma_{N}=75 \mu \mathrm{Jy}$ beam ${ }^{-1}$ is the rms measured at the location of the north component. The right panel corresponds to a zoom-in of the image centered at the location of the south component SR 24S.
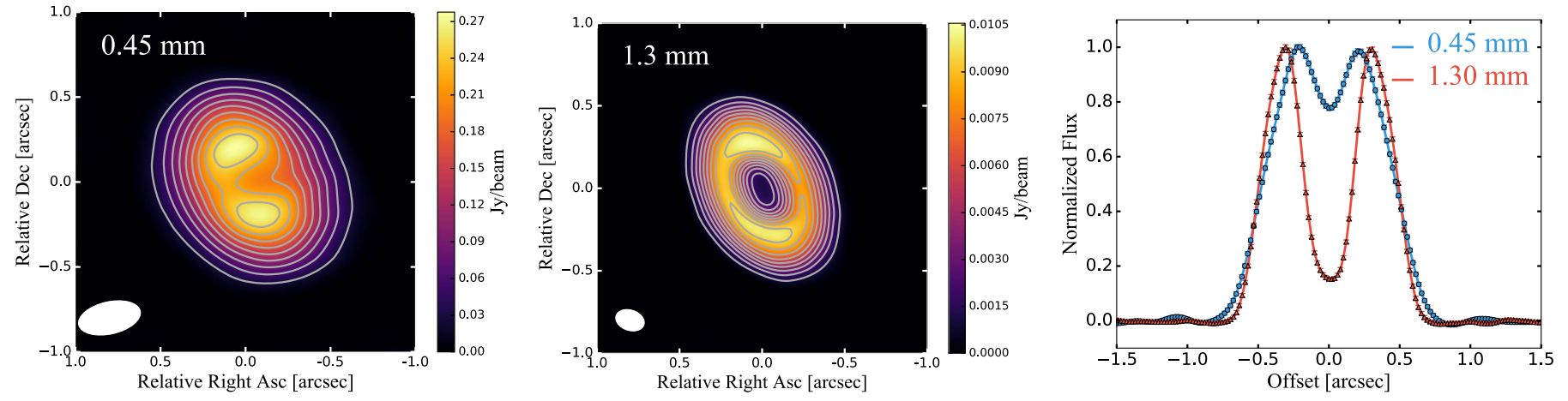

Figure 2. Continuum observations of the transition disk SR 24S. Left panel: ALMA continuum map at $0.45 \mathrm{~mm}$. Middle panel: ALMA continuum map at $1.3 \mathrm{~mm}$. Specific details are summarized in Table 1 . For both maps, the contour levels are $10 \%, 20 \%, \ldots, 90 \%$ of the peak of emission. Right panel: normalized continuum flux at 0.45 and $1.3 \mathrm{~mm}$ along the $\mathrm{PA}=24^{\circ}$ of the disk (error bars are also included, which are of the size of the points).

mass can be estimated as (Hildebrand 1983)

$$
M_{\mathrm{dust}} \simeq \frac{d^{2} F_{\nu}}{\kappa_{\nu} B_{\nu}(T(r))}
$$

where $d$ is the distance to the source, $\kappa_{\nu}$ is the dust opacity at a given frequency, and $B_{\nu}(T)$ is the Planck function for a given temperature radial profile $T(r)$. Assuming a distance of $137 \mathrm{pc}$, a dust opacity at $1.3 \mathrm{~mm}$ of $\sim 3 \mathrm{~cm}^{2} \mathrm{~g}^{-1}$ (e.g., Andrews et al. 2011) and a temperature of $20 \mathrm{~K}$, the upper limit for the dust mass of the SR $24 \mathrm{~N}$ disk is $M_{\text {dust,SR } 24 \mathrm{~N}} \lesssim 3.5 \times 10^{-7} M_{\odot}$ or in Earth masses equivalent to $\lesssim 0.12 M_{\oplus}$.
Table 1

Properties of the Continuum ALMA Images of SR 24S (Figure 2)

\begin{tabular}{lccccc}
\hline \hline Cycle & $\begin{array}{c}\text { beam } \\
\left({ }^{\prime \prime}\right)\end{array}$ & $\begin{array}{c}\lambda \\
(\mathrm{mm})\end{array}$ & $\begin{array}{c}F_{\text {peak }} \\
(\mathrm{mJy})\end{array}$ & $\begin{array}{c}F_{\text {total }} \\
(\mathrm{mJy})\end{array}$ & $\begin{array}{c}\sigma \\
\left(\mathrm{mJy} \mathrm{beam}^{-1}\right)\end{array}$ \\
\hline 0 & $0.37 \times 0.19$ & 0.45 & 278 & 1885 & 1.9 \\
\hline 2 & $0.18 \times 0.12$ & 1.30 & 15.4 & 220 & 0.06 \\
\hline
\end{tabular}

Figure 2 presents the continuum ALMA observations of SR 24S at $0.45 \mathrm{~mm}$ and at $1.3 \mathrm{~mm}$. The details of the calibration process for the Cycle 0 data are presented in Pérez 

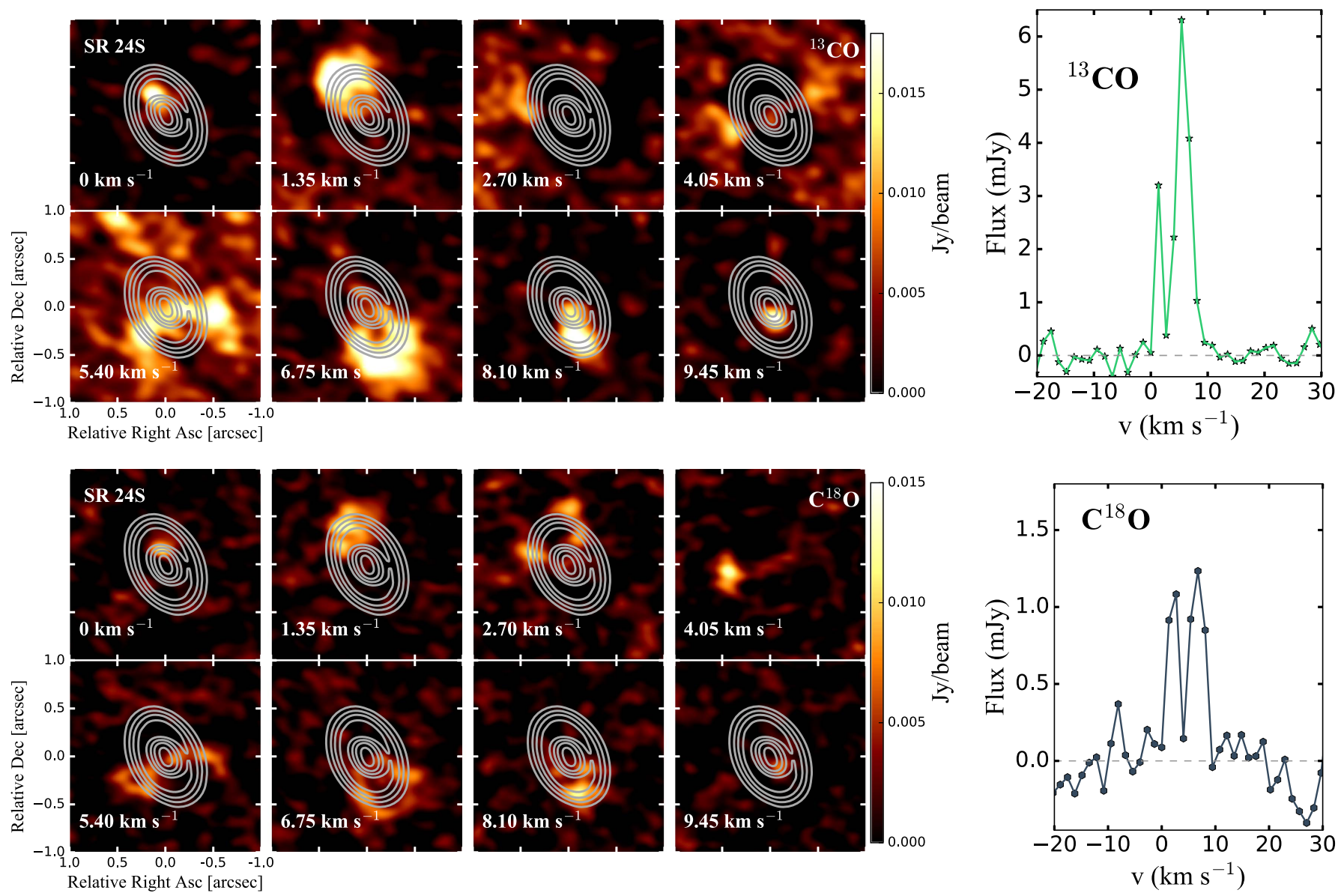

Figure 3. Left panels: channel maps of the ${ }^{13} \mathrm{CO}$ (top panels) and $\mathrm{C}^{18} \mathrm{O}$ (bottom panels) emission in SR $24 \mathrm{~S}$ from 0 to $9.45 \mathrm{~km} \mathrm{~s}{ }^{-1}$. The spectral resolution is $1.35 \mathrm{~km} \mathrm{~s}^{-1}$ and the rms is around $1.4 \mathrm{mJy}_{\text {beam }}^{-1}$ per channel for both lines. The contour levels are $20 \%, \ldots, 100 \%$ of the peak of the dust-continuum emission. Right panels: ${ }^{13} \mathrm{CO}$ and $\mathrm{C}^{18} \mathrm{O}(J=2-1)$ spectrum by integrating over a circular area centered at the location of SR $24 \mathrm{~S}$ and with a radius of $1^{\prime \prime}$.

et al. (2014). We summarize the properties of each image in Table 1. The continuum emission is detected with a signal-tonoise ratio (with respect to the peak) of 146 for the $0.45 \mathrm{~mm}$ data and 256 for the $1.3 \mathrm{~mm}$ data (see Table 1). The total flux at $0.45 \mathrm{~mm}$ is $1.9 \mathrm{Jy}$ and at $1.3 \mathrm{~mm}$ it is $0.22 \mathrm{Jy}$. Calculating the dust disk mass from $1.3 \mathrm{~mm}$ flux, and assuming the same opacity and temperature as for SR $24 \mathrm{~N}$, we obtain that $M_{\text {dust,SR 24S }} \sim 3.0 \times 10^{-4} M_{\odot}$, implying a dust disk ratio between the southern and the northern component of $\gtrsim 840$. Nonetheless, Equation (1) assumes optically thin emission, which may not be the case for SR $24 \mathrm{~S}$, especially close to the location of the ring (see. Section 4.2). If only part of the emission is optically thin, the dust mass for the disk around SR 24S is underestimated, which increases the dust mass disk ratio between the southern and the northern components.

Taking the total flux at each wavelength, the integrated spectral index is given by $\alpha_{\mathrm{mm}}=\ln \left(F_{1.3 \mathrm{~mm}} / F_{0.45 \mathrm{~mm}}\right) /$ $\ln (0.45 \mathrm{~mm} / 1.3 \mathrm{~mm})=2.02 \pm 0.13$ (the error includes a calibration uncertainty of $10 \%$ ), which is lower than the value previously reported (Pinilla et al. 2014) based on SMA and ATCA observations at 0.88 and $3.0 \mathrm{~mm}$ (Ricci et al. 2010; Andrews et al. 2011). This low value may indicate grain growth and/or a small cavity, but most likely arises from optically thick emission as discussed in Section 4.2.

Figure 2 also shows the continuum flux normalized to the peak of emission at 0.45 and $1.3 \mathrm{~mm}$ of a radial cut along the PA of the disk (obtained in Section 4). Both profiles reveal a cavity and a ring. The $1.3 \mathrm{~mm}$ emission strongly decreases inside the cavity where the flux is reduced by around $85 \%$. In contrast, the $0.45 \mathrm{~mm}$ emission shows a shallower cavity, with the emission reduced by about $24 \%$ compared to the peak of emission. In addition, the position of the peak of the ring is located further out at $1.3 \mathrm{~mm}$. However, this contrast and location of the cavity can be affected by the beam convolution and a more detailed analysis of the intensity profiles is done in the visibility domain in Section 4.1.

\subsection{Gas emission}

Both ${ }^{13} \mathrm{CO}$ and $\mathrm{C}^{18} \mathrm{O}(J=2-1)$ lines are detected, but are affected by foreground absorption from the nearby dark cloud. In particular ${ }^{13} \mathrm{CO}$ is more contaminated than $\mathrm{C}^{18} \mathrm{O}$ because $\mathrm{C}^{18} \mathrm{O}$ is more optically thin than ${ }^{13} \mathrm{CO}$. Figure 3 shows the channel maps of the ${ }^{13} \mathrm{CO}$ and $\mathrm{C}^{18} \mathrm{O}$ emission in SR $24 \mathrm{~S}$ from 0 to $9.45 \mathrm{~km} \mathrm{~s}^{-1}$. In addition, the ${ }^{13} \mathrm{CO}$ and $\mathrm{C}^{18} \mathrm{O}(J=2-1)$ spectrum is shown in Figure 3, which is obtained by integrating over a circular area centered at the location of SR $24 \mathrm{~S}$ and with a radius of $1^{\prime \prime}$. These channel maps confirm the presence of ${ }^{13} \mathrm{CO}$ and $\mathrm{C}^{18} \mathrm{O}$ in the SR $24 \mathrm{~S}$ disk, but also the effect of the foreground absorption, in particular in the channels of 2.7 and $4.05 \mathrm{~km} \mathrm{~s}^{-1}$. Thus, the asymmetry of the double-peaked velocity profile for both lines is likely due to this foreground absorption.

Figure 4 shows the zero-moment map for ${ }^{13} \mathrm{CO}$ and $\mathrm{C}^{18} \mathrm{O}$ obtained from $-1.35 \mathrm{~km} \mathrm{~s}^{-1}$ to $10.8 \mathrm{~km} \mathrm{~s}^{-1}$, where the 

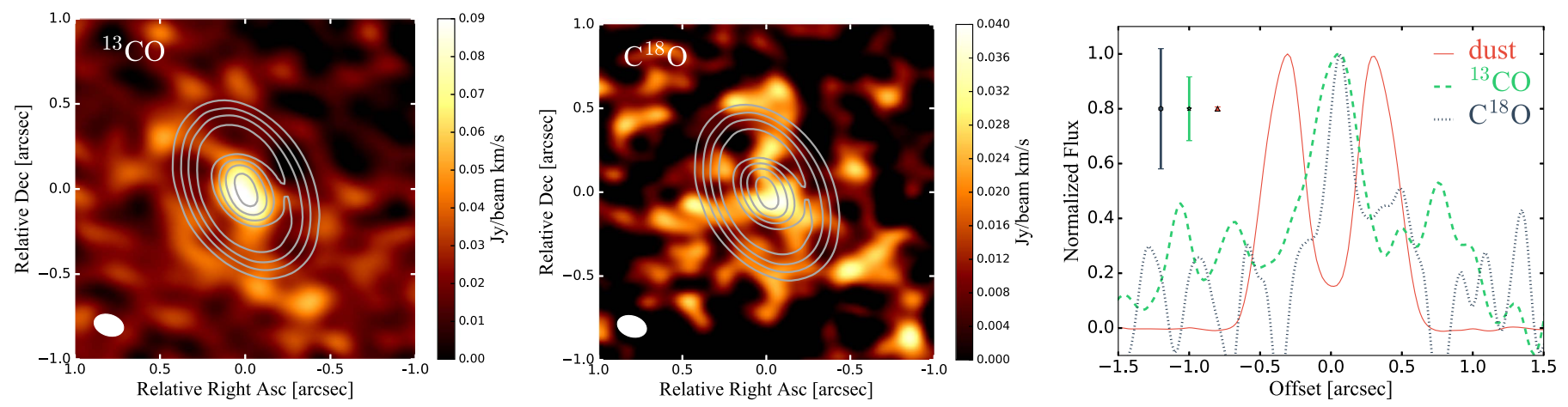

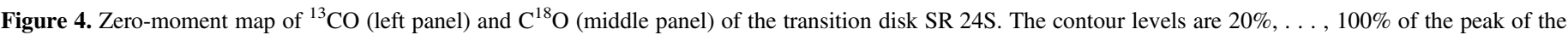

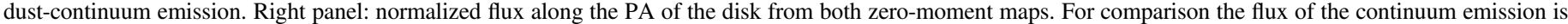
overplotted.

channels contain significant emission $(\gtrsim 5 \sigma$, with $\sigma=1.4$ mJy beam ${ }^{-1}$ per $1.35 \mathrm{~km} \mathrm{~s}^{-1}$ channel). For ${ }^{13} \mathrm{CO}$, inside a circle of $\sim 0$." 7 radius centered at the location of SR $24 \mathrm{~S}$, the total flux per velocity interval is $\sim 1 \mathrm{Jy} \mathrm{beam}^{-1} \mathrm{~km} \mathrm{~s}^{-1}$, and approximately $60 \%$ of this emission comes from the inner part of the disk inside the millimeter dust cavity. The emission peaks at the center with a value of $\sim 0.1 \mathrm{Jy}_{\text {beam }}{ }^{-1} \mathrm{~km} \mathrm{~s}^{-1}$ and the rms of the zero-moment map is around $\sim 10 \mathrm{mJy}_{\text {beam }}^{-1} \mathrm{~km} \mathrm{~s}^{-1}$, which gives a signal-tonoise ratio of 10 with respect to the peak and 100 with respect to the total flux. For $\mathrm{C}^{18} \mathrm{O}$, inside the same circle of $\sim 0$ " 7 radius, the total flux per velocity is $\sim 0.5 \mathrm{Jy} \mathrm{beam}^{-1} \mathrm{~km} \mathrm{~s}^{-1}$, and approximately $40 \%$ of this emission comes from the inner part. This emission also peaks at the center with a value of $\sim 40 \mathrm{mJy}^{\text {beam }}{ }^{-1} \mathrm{~km} \mathrm{~s}^{-1}$ and the rms of the zero-moment map is around $\sim 8.6 \mathrm{mJy}^{\text {beam }}{ }^{-1} \mathrm{~km} \mathrm{~s}^{-1}$, which gives signal-to-noise ratios of 5 and 58 for the peak and the total flux, respectively.

The flux normalized to the peak of the ${ }^{13} \mathrm{CO}$ and the $\mathrm{C}^{18} \mathrm{O}$ in a radial cut along the PA of the disk is also shown in Figure 4. For comparison, the normalized flux of the continuum millimeter emission is overplotted. The peak of both lines resides inside the millimeter cavity. The uncertainties for the flux are also shown and therefore the wiggles of emission beyond 0.44 of ${ }^{13} \mathrm{CO}$ and $\mathrm{C}^{18} \mathrm{O}$ are within the noise of the observations and they are not significant. We do not derive the inclination and PA from the gas emission, but from the dust-continuum emission. Moreover, because of the foreground absorption in some of the channels, we do not estimate gas masses from the current observations of CO isotopologues as done by e.g., Williams \& Best (2014) and Miotello et al. (2016).

In the northern component of the SR 24 system (SR 24N), there is no significant detection of ${ }^{13} \mathrm{CO}$ or $\mathrm{C}^{18} \mathrm{O}$ (i.e., nothing $\gtrsim 3 \sigma$, where $\sigma$ has a value of around $\sim 1.5 \mathrm{mJy}$ beam $^{-1}$ per $1.35 \mathrm{~km} \mathrm{~s}^{-1}$ channel).

\section{Data Analysis}

\subsection{Disk Morphology}

All the following analysis for fitting the morphology of the disk from the dust-continuum emission is performed in the visibility domain for the two wavelengths separately. We work with each observed $(u, v)$ point since we do not assume any a priori knowledge of the total flux, inclination, position angle, and center of the image. Hence, these are free parameters of each of the explored models ( $F_{\text {total }}, i$, PA, $x_{0}$ and $y_{0}$, being $x_{0}$ and $y_{0}$ the potential offset from the center taken at $\alpha_{2000}=16: 26: 58.5$, $\delta_{2000}=-24: 45: 37.2$ ). As a first approximation for the structure, we assume an axisymmetric disk (Figure 2), and thus we focus on fitting the real part of the visibilities. The Fourier transform of a symmetric brightness distribution can be expressed in terms of the zeroth-order Bessel function of the first kind $J_{0}$ of the deprojected uv-distance, such that

$$
V_{\text {Real }}\left(r_{u v}\right)=2 \pi \int_{0}^{\infty} I(r) J_{0}\left(2 \pi r_{u v} r\right) r d r
$$

where $r_{u v}=\sqrt{u_{\phi}^{2} \cos i^{2}+v_{\phi}^{2}}$, with $u_{\phi}=u \cos \phi+v \sin \phi$ and $v_{\phi}=-u \sin \phi+v \cos \phi$, and $i$ and $\phi$ being the inclination and position angle of the disk, respectively.

To fit the morphology of the disk, because the visibilities and continuum maps reveal a cavity at the two wavelengths, we explore models where the intensity profile has a ring shape. The fitting is conducted using the Markov chain Monte Carlo (MCMC) method. The first model we use is a radially symmetric Gaussian ring, with two extra free parameters, for a total of seven free parameters $\left(R_{\text {peak }}, R_{\text {width }}, F_{\text {total }}, i, \mathrm{PA}, x_{0}\right.$ and $\left.y_{0}\right)$, such that the intensity radial profile is given by

$$
I(r)=C \exp \left(-\frac{\left(r-R_{\text {peak }}\right)^{2}}{2 R_{\text {width }}^{2}}\right),
$$

where the constant $C$ is related with the total flux of the disk as

$$
C=\frac{F_{\text {total }}}{\int_{0}^{\infty} I(r) J_{0}(0) r d r} .
$$

The cuts along the PA of the disk in Figure 2 show that the ring is not necessarily a symmetric Gaussian around the peak in the radial direction (in particular for the $0.45 \mathrm{~mm}$ emission), and hence we use two other different models to mimic a radially asymmetric ring (still azimuthally symmetric since our models are focused on fitting the real part of the visibilities). In the first of these models we assume an asymmetric Gaussian with two different widths that coincide at the location of the peak of emission, such that

$$
I(r)=\left\{\begin{array}{l}
C \exp \left(-\frac{\left(r-R_{\text {peak }}\right)^{2}}{2 R_{\text {width }}^{2}}\right) \text { for } r \leqslant R_{\text {peak }} \\
C \exp \left(-\frac{\left(r-R_{\text {peak }}\right)^{2}}{2 R_{\text {width2 }}^{2}}\right) \text { for } r>R_{\text {peak }} .
\end{array}\right.
$$

This model has a total of eight free parameters: $R_{\text {peak }}, R_{\text {width }}$, $R_{\text {width2 }}, F_{\text {total }}, i$, PA, $x_{0}$, and $y_{0}$. This radially asymmetric ring model is also motivated by the results of particle trapping in radial pressure bumps. These models of dust evolution predict 
that the regions where dust accumulates become narrower for larger grains (and therefore for longer wavelengths). Additionally, it is expected that the accumulation is radially narrower at longer times of evolution ( $~ \gtrsim 1 \mathrm{Myr}$ ), because micron-sized dust particles require more time to grow to millimeter sizes in the outer parts of the disk, from where they will then drift toward the pressure bump. At longer times ( $~ 5 \mathrm{Myr})$, the emission from the models is expected to be a symmetric ring. As a consequence, the morphology of the trapped dust is expected to be an asymmetric ring in the radial direction (which can be mimicked assuming $R_{\text {width }}<R_{\text {width2 }}$ in Equation (5)) at shorter times after the pressure bump is formed ( $\lesssim 1 \mathrm{Myr}$ ), becoming narrower and radially symmetric at longer times ( $~ 5 \mathrm{Myr}$; see, e.g., Figure 4 from Pinilla et al. 2015).

Second, we assume a combination of a Gaussian profile with a power law. This also has eight free parameters, namely $R_{\text {peak }}, R_{\text {width }}, \gamma, F_{\text {total }}, i, \mathrm{PA}, x_{0}$, and $y_{0}$, given by

$$
I(r)=C\left[r^{-\gamma}+\exp \left(-\frac{\left(r-R_{\text {peak }}\right)^{2}}{2 R_{\text {width }}^{2}}\right)\right] .
$$

The motivation of this model is to investigate the potential emission from the inner disk and its possible dependency with wavelength.

For the fits, we used emcee (Foreman-Mackey et al. 2013), which allows us to efficiently sample the parameter space in order to maximize the likelihood result for each model. Maximizing the likelihood function $(\mathscr{L}(\Theta))$ is equivalent to minimizing the negative of the logarithm of the likelihood, since the logarithm is an increasing function over the entire range. Therefore we aim to minimize the following function:

$$
\begin{aligned}
-\log (\mathscr{L}(\Theta))= & -\frac{1}{2} \sum_{i=1}^{n}\left[\log \left(2 \pi \sigma_{i}^{2}\right)\right. \\
& \left.+\frac{\left(V_{\text {Real,obs }}^{i}-V_{\text {Real,model }}^{i}\right)^{2}}{2 \sigma_{i}^{2}}\right],
\end{aligned}
$$

where $\sigma$ is the uncertainty of each observed $(u, v)$ point, $n$ is the total number of data, $V_{\text {Real,obs }}$ is the real part of the observed visibilities, and $V_{\text {Real,model }}$ are the visibilities for each model calculated with Equation (2). We adopted a set of uniform prior probability distributions for the free parameters explored by the Markov chain in the three models, specifically:

$$
\begin{aligned}
R_{\text {peak }} & \in[1,100] \mathrm{au} \\
R_{\text {width }} & \in[1,50] \mathrm{au} \\
R_{\text {width } 2} & \in[1,50] \mathrm{au} \\
F_{\text {total }} & \in[1.0,5.0] \mathrm{Jy} \quad \text { for the } 0.45 \mathrm{~mm} \text { data } \\
F_{\text {total }} & \in[0.02,2.5] \mathrm{Jy} \text { for the } 1.3 \mathrm{~mm} \text { data } \\
i & \in[10,80]^{\circ} \\
\mathrm{PA} & \in[10,80]^{\circ} \\
x_{0} & \in[-0.2,0.2]^{\prime \prime} \\
y_{0} & \in[-0.2,0.2]^{\prime \prime} \\
\gamma & \in[-3,3] .
\end{aligned}
$$

For the radial grid, we assume $r \in$ [1-500] au with steps of $0.1 \mathrm{au}$. The burn-in phase for convergence is $\sim 1000$ steps, which is $\sim 10$ times the autocorrelation time of 100 steps (e.g., Sokal 1994; Tazzari et al. 2016). We let the Markov chain sample the parameter space for another thousands of steps, for a total of 4000 steps with 1000 walkers. Each measurement set is fitted separately and therefore we used emcee to fit a total of six models (three models for two different data sets). To simplify the fitting process, we obtained the PA and the $i$ using the $1.3 \mathrm{~mm}$ data (since it has better signal-to-noise) for each model, and keep the best-fit values of these two parameters to fit the $0.45 \mathrm{~mm}$ data.

The results are summarized in Tables 2-4 and Figure 6. All three models show that the peak of the ring is located farther out and becomes narrower at longer wavelength, with a difference of around $\sim 20$ au for the location of the peak and $\sim 10$ au for the width(s). The models of the radially asymmetric ring show that $R_{\text {width }}<R_{\text {width2, creating a slightly (radially) }}$ asymmetric ring with an outer tail. The model of the power law together with a Gaussian gives a slightly steeper profile for the $0.45 \mathrm{~mm}$ emission. The inclination and position angle obtained with the three models give very similar values, with mean values of $46^{\circ}$ and $24^{\circ}$, respectively, in agreement with the values found by Andrews et al. (2011) and van der Marel et al. (2015), and those in Section 2. The values obtained for the shift of the center are very low compared with the pixel sizes from the observations $(0$ !" 02 for the $1.3 \mathrm{~mm}$ observations and 0 ". 04 for the $0.45 \mathrm{~mm}$ observations).

We image the models and residuals (data-model) using identical $(u, v)$ coordinates as the actual ALMA observations. Figure 5 shows the synthetic images of the best-fit models and the corresponding residuals. In general, the quality of the three fits is similar. For the $1.3 \mathrm{~mm}$ data, all three models reproduce roughly the same amount of residuals with respect to the rms of the observations. This is because all three models resample an almost symmetric ring and a quite empty cavity, where the intensity decreases around $80 \%-90 \%$ with respect to the peak of emission, similar to the observations (left panel Figure 2). For the $0.45 \mathrm{~mm}$ data, the asymmetric ring is the model that reproduces less residuals, where the emission inside the cavity only decreases by $\sim 20 \%$ with respect to the peak of emission.

Figure 6 shows the profile of the normalized intensity with respect to peak value, calculated with the best-fit parameters (Tables 2-4), for each case and each wavelength (for this plot, the inclination and the position angle are taken to have the same value for all three models, that is, the mean value, $i=46^{\circ}$ and $\mathrm{PA}=24^{\circ}$ ). These three models resemble a roughly symmetric ring-like emission at the two wavelengths, and the profiles in Figure 6 are comparable with the azimuthally averaged radial profile of the deprojected images (left panel in Figure 7).

Independently of the model, an asymmetric structure persists in the maps of the residuals (Figure 5). The model underpredicts the flux in the northeast and overpredicts the flux in the southwest with similar magnitude and morphology. The residuals show a spiral-shape-like structure similar to those found in the residual maps of the visibilities analysis of SR 21 and HD 135344B (Pérez et al. 2014; Pinilla et al. 2015). The residuals peak along $\sim 47 \pm 2$. Taking a radial cut along this angle, the positive residuals peak around $0.45 \pm 0$." 1 from the center, and the dip of emission has its minimum around the same location in the opposite side of the disk. The residual map of the $1.3 \mathrm{~mm}$ data shows an additional structure at $\sim 0$." 15 with a swap in the flux emission (negative in the northeast and positive in the southwest). As an experiment, we also performed the MCMC fitting keeping PA, $i, x_{0}$, and $y_{0}$ fixed and assuming the values obtained with uvmodelfit. In this 
Table 2

Results from the MCMC Fitting ALMA Data

\begin{tabular}{|c|c|c|c|c|c|c|c|}
\hline Data & $R_{\text {peak }}(\mathrm{au})$ & $R_{\text {width }}(\mathrm{au})$ & $F_{\text {total }}(\mathrm{Jy})$ & $i\left(^{\circ}\right)$ & $\mathrm{PA}\left({ }^{\circ}\right)$ & $x_{0}$ (mas) & $y_{0}$ (mas) \\
\hline $0.45 \mathrm{~mm}$ & $20.66_{-3.17}^{+3.25}$ & $36.62_{-2.49}^{+2.36}$ & $1.85_{-0.16}^{+0.16}$ & fixed & fixed & $-3.17_{-0.17}^{+0.30}$ & $2.90_{-0.34}^{+0.16}$ \\
\hline $1.30 \mathrm{~mm}$ & $42.15_{-2.67}^{+2.29}$ & $21.41_{-2.04}^{+2.69}$ & $0.21_{-0.02}^{+0.01}$ & $46.17_{-1.02}^{+2.78}$ & $24.73_{-1.31}^{+3.16}$ & $-1.40_{-0.97}^{+0.45}$ & $2.64_{-0.78}^{+0.33}$ \\
\hline
\end{tabular}

Note. Ring model (Equation (3)).

Table 3

Results from the MCMC Fitting ALMA Data

\begin{tabular}{|c|c|c|c|c|c|c|c|c|}
\hline Data & $R_{\text {peak }}(\mathrm{au})$ & $R_{\text {width }}(\mathrm{au})$ & $R_{\text {width2 }}(\mathrm{au})$ & $F_{\text {total }}(\mathrm{Jy})$ & $i\left(^{\circ}\right)$ & $\mathrm{PA}\left({ }^{\circ}\right)$ & $x_{0}$ (mas) & $y_{0}$ (mas) \\
\hline $0.45 \mathrm{~mm}$ & $20.88_{-3.02}^{+2.06}$ & $28.81_{-2.54}^{+3.56}$ & $35.77_{-1.57}^{+1.32}$ & $1.83_{-0.16}^{+0.19}$ & fixed & fixed & $-2.57_{-0.08}^{+0.07}$ & $1.95_{-0.45}^{+0.11}$ \\
\hline $1.30 \mathrm{~mm}$ & $37.45_{-2.91}^{+2.85}$ & $16.46_{-2.94}^{+3.96}$ & $25.00_{-1.67}^{+1.13}$ & $0.21_{-0.02}^{+0.01}$ & $45.76_{-0.87}^{+2.87}$ & $23.63_{-0.61}^{+2.56}$ & $-0.54_{-0.05}^{+0.08}$ & $1.63_{-0.34}^{+0.14}$ \\
\hline
\end{tabular}

Note. Radially asymmetric ring (Equation (5)).

Table 4

Results from the MCMC Fitting ALMA Data

\begin{tabular}{llrrrrrrr}
\hline \hline Data & $R_{\text {peak }}(\mathrm{au})$ & $R_{\text {width }}(\mathrm{au})$ & $\gamma$ & $F_{\text {total }}(\mathrm{Jy})$ & $i\left(^{\circ}\right)$ & PA $\left(^{\circ}\right)$ & $x_{0}(\mathrm{mas})$ \\
\hline $0.45 \mathrm{~mm}$ & $21.29_{-1.62}^{+2.27}$ & $36.06_{-1.25}^{+1.51}$ & $1.64_{-0.29}^{+0.27}$ & $1.87_{-0.17}^{+0.19}$ & fixed & fixed & $-4.44_{-0.54}^{+0.23}$ & $2.85_{-0.76}^{+0.32}$ \\
\hline $1.30 \mathrm{~mm}$ & $43.98_{-1.46}^{+1.79}$ & $22.74_{-1.96}^{+2.91}$ & $1.34_{-0.29}^{+0.24}$ & $0.21_{-0.01}^{+0.02}$ & $46.31_{-1.07}^{+1.88}$ & $24.30_{-0.86}^{+2.14}$ & $-1.21_{-0.45}^{+0.28}$ & $1.47_{-0.53}^{+0.39}$ \\
\hline
\end{tabular}

Note. Power law + ring-Gaussian (Equation (6)). The range given for each parameter corresponds to the 95\% credible range. The PA and $i$ are fixed for the fitting of $0.45 \mathrm{~mm}$ data and are taken to be the same values found from the fitting of the $1.3 \mathrm{~mm}$ data (specifically PA $=24^{\circ}$ and $i=46^{\circ}$ ).
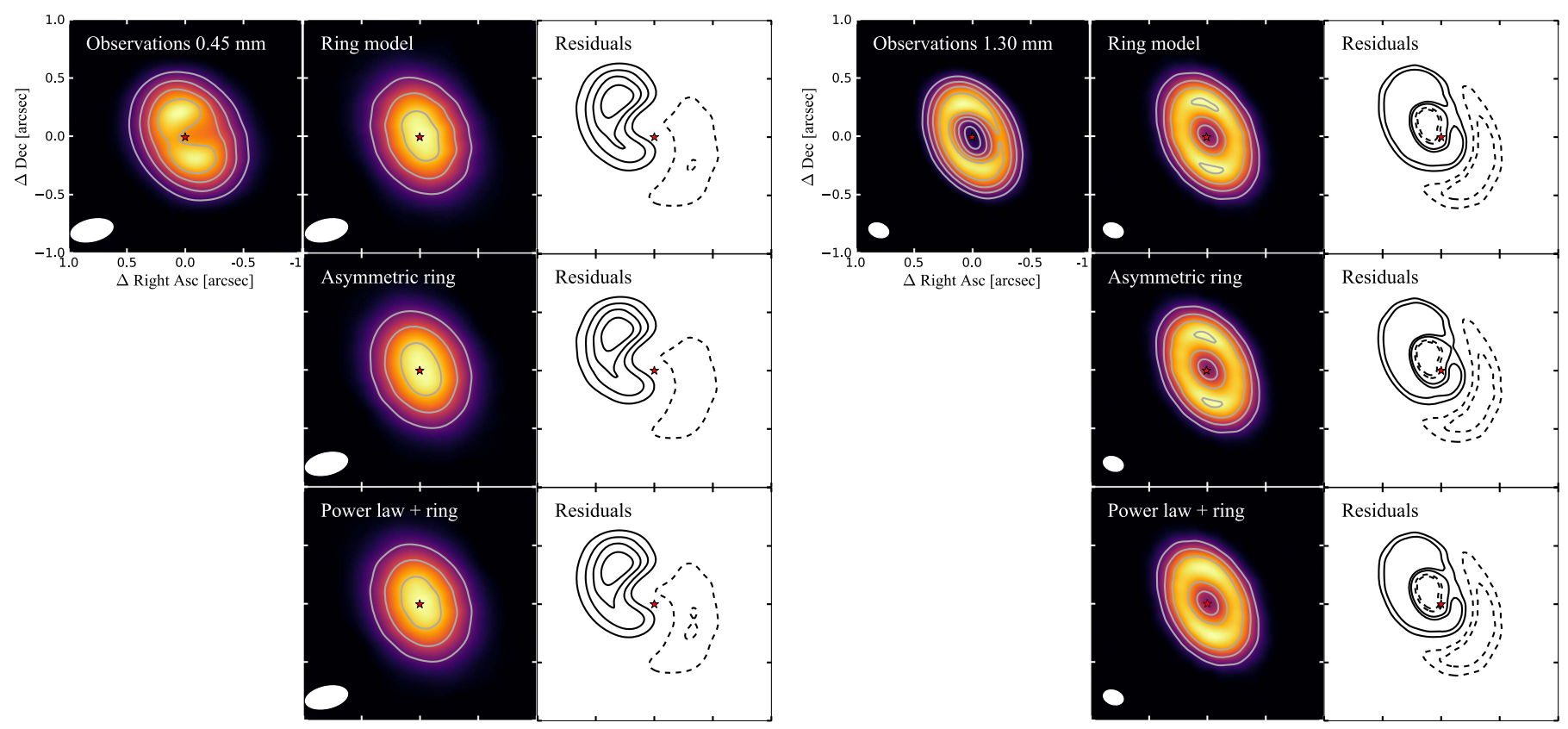

Figure 5. Observations, best-fit models, and residuals for the $0.45 \mathrm{~mm}$ data (left panels) and the $1.3 \mathrm{~mm}$ data (right panels). In the observations and model maps the contours are every $20 \%, \ldots, 100 \%$ of the peak value (Table 1 ). In the residual maps (data model), the contours are every $\pm 3 \sigma$ (from $-12 \sigma$ to $12 \sigma$ for the $0.45 \mathrm{~mm}$ model and from $-6 \sigma$ to $6 \sigma$ for the $1.3 \mathrm{~mm}$ model), showing the negative contours with dashed lines.

experiment, we found a higher amount of residuals, but with similar shape as the ones shown in Figure 5. In our analysis, we only fitted the real part of the visibilities, assuming none azimuthal variations. As a result, if an asymmetry arises due to an offset, the model optimizes the fit toward a symmetric emission. Hence, in the framework of our models, it is difficult 

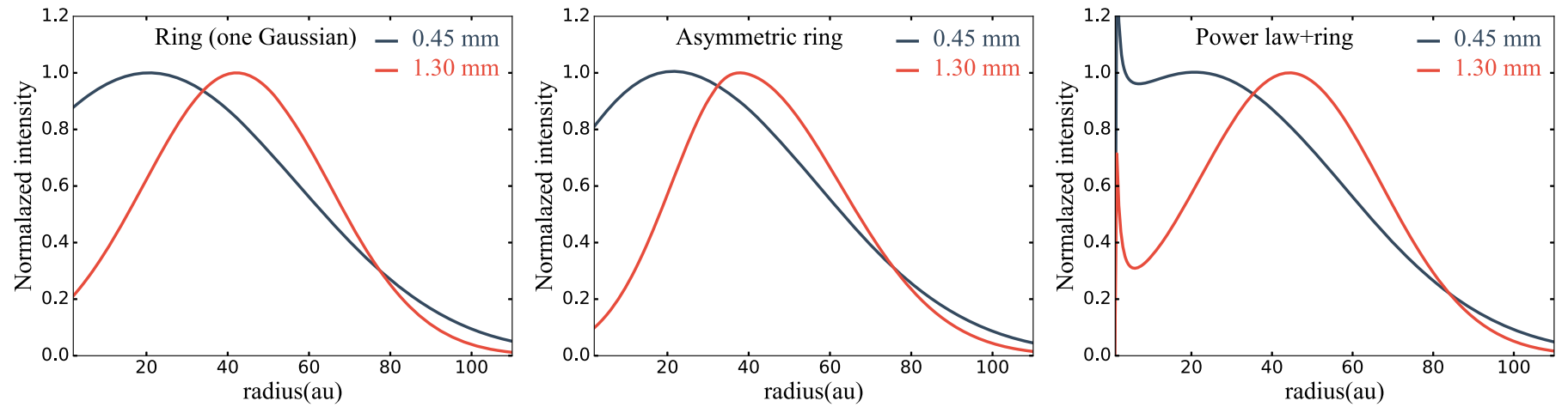

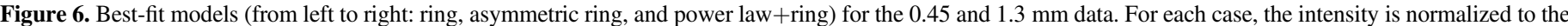
value at the location of the peak of the ring.
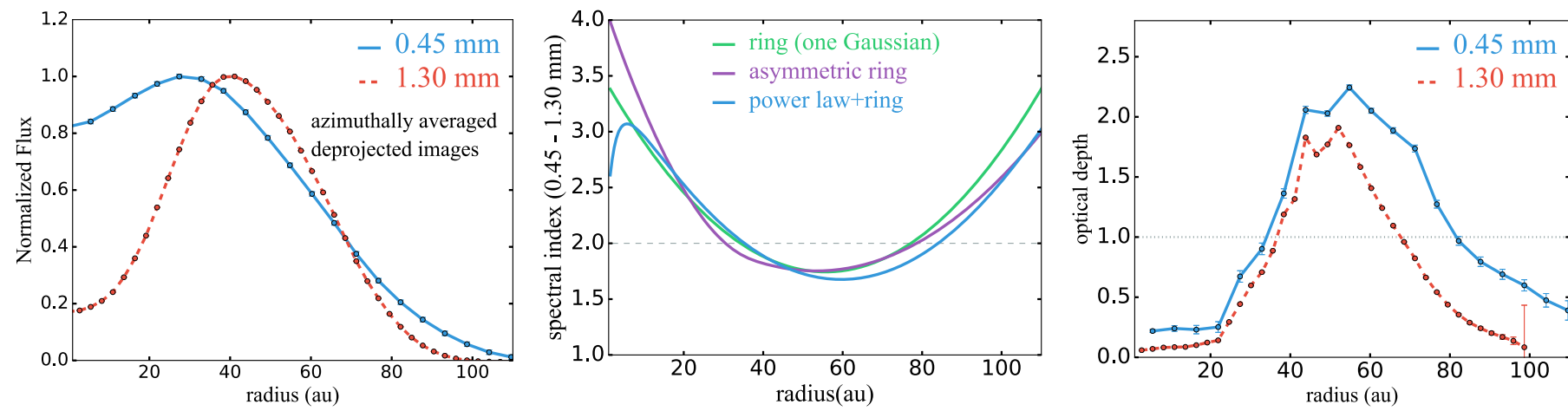

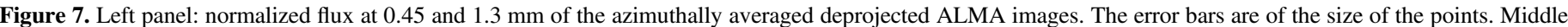

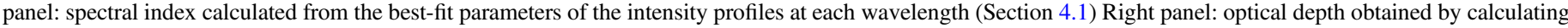

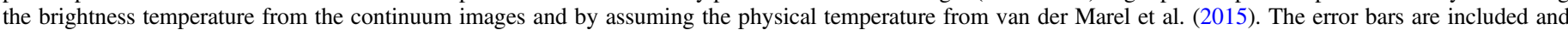
obtained from error propagation, taking into account the rms and the standard deviation from the azimuthally averaged flux.

to conclude how significant is the amount of residuals, and their shapes and higher angular resolution observations are required to confirm if these potential asymmetries are real.

Zhang et al. (2016) modeled the visibility profile of several disks with multiple rings (such as HL Tau, TW Hya, and HD 163296). Applying their method to our data does not seem suitable since there is not more than one distinctive peak in the visibility profile. However, as a test we did an experiment of fitting the visibilities at $1.3 \mathrm{~mm}$ with at least two rings. In this case, the fit converges to one single ring that dominates the intensity profile. More complex models that include asymmetric structures, such as spiral arms, may improve the fit (Figure 5). However, due to the high degeneracy of including several parameters to fit both the imaginary and the real part of the visibilities simultaneously, we do not perform such analysis.

\subsection{Optical Thickness and Spectral Index Interpretation}

The slope of the spectral energy distribution (SED) at millimeter wavelengths, or spectral index $\left(\alpha_{\mathrm{mm}}\right.$, such that $\left.F_{\mathrm{mm}} \propto \nu^{\alpha} \mathrm{mm}\right)$, has been widely used to trace millimeter grains in protoplanetary disk. If the millimeter emission is optically thin, low values of the spectral index $(\lesssim 3.5)$ indicate the growth of particles to millimeter sizes. The spatially integrated spectral index $\left(\alpha_{\mathrm{mm}}\right)$ in protoplanetary disks observed in different starforming regions and around different stellar types has values lower than 3.5 (e.g., Birnstiel et al. 2010; Testi et al. 2014). Spatial variations of the spectral index in different protoplanetary disks have been resolved, where in most of the cases the spectral index increases radially, evidencing that the grain size decreases for increasing radius (e.g., Banzatti et al. 2011; Pérez et al. 2012, 2015; Trotta et al. 2013; Tazzari et al. 2016), as expected from radial drift, as seen in dust evolution models (e.g., Birnstiel et al. 2012).

However, for transition disks the spectral index is expected to increase toward the outer edge of the cavity, that is, toward the location where particles are trapped and have grown to millimeter sizes. For these disks, the spatially integrated spectral index is also expected to be higher for larger cavities (Pinilla et al. 2014). There are few transition disks where the spectral index has been imaged, HD 142527, IRS 48, and SR 21 (Casassus et al. 2015; Pinilla et al. 2015; van der Marel et al. 2015), and in these few cases the spectral index decreases toward the location where the millimeter emission peaks and where a particle accumulation is expected.

The middle panel of Figure 7 shows the radial profile of the spectral index calculated from the intensity profiles taking the best-fit parameters for each model at each wavelength described in Section 4.1. At the location of the ring, the spectral index has values lower than 2.0, specifically from $32 \pm 3$ au to $81 \pm 4$ au. The right panel of Figure 7 also shows the optical depth $\tau$ obtained from the brightness temperature, which is calculated from the azimuthally averaged flux of the deprojected image (displayed in the left panel of Figure 7) and without assuming the Rayleigh-Jeans regime. For the physical temperature, we assume the midplane values from the dust 
radiative transfer models for SR 24S in van der Marel et al. (2015). The error bars are obtained from error propagation, taking into account the rms of the observations and the standard deviation from the azimuthally averaged flux values. With the assumed temperature, the emission is optically thick at both wavelengths $(\tau>1)$ close to the location of the ring-like emission. In particular, $\tau>1$ from $\sim 33$ to $\sim 81$ au for the $0.45 \mathrm{~mm}$ emission and from $\sim 36$ to $\sim 66$ au for the $1.3 \mathrm{~mm}$ emission. Therefore, the decrease of the spectral index toward the location of the ring is likely because of optical thickness and with the current observations, $\alpha_{\mathrm{mm}}$ cannot be interpreted as grain growth inside the ring.

Observations at shorter (optically thick) wavelengths can provide information of the temperature distribution in SR 24S; and at longer (optically thin) wavelengths, which can give direct information of the dust size, are therefore needed to better constrain the dust density distribution in SR $24 \mathrm{~S}$.

\section{Discussion}

Embedded planets inside the millimeter cavities are commonly used to explain the observed gas and dust structures of transition disks. From planet-disk interaction together with dust evolution models, it is expected that at the outer edge of the planet-carved gap, the dust particles accumulate and grow to millimeter sizes. For the gas, it is expected that the cavity is smaller and less depleted than the millimeter cavity, as observed in several transition disks (e.g., van der Marel et al. 2016). The small grains (micron-sized) particles are also expected to have a different distribution than the millimeter dust, with smaller or no gaps, depending on planet mass and disk viscosity (e.g., de Juan Ovelar et al. 2016).

From the current observations of ${ }^{13} \mathrm{CO}$ and $\mathrm{C}^{18} \mathrm{O}$ in the transition disk SR 24S, the gas emission is centrally peaking inside the millimeter dust cavity. It is possible that the gas cavity (or gap) remains unresolved or that the gas is not depleted inside the millimeter cavity. The latter case suggests that if embedded planets are the cause of the millimeter cavity, they must be low-mass planets $\left(\lesssim 0.1-1 M_{\text {Jup }}\right.$; see, e.g., Pinilla et al. 2012b; Zhu et al. 2012; Rosotti et al. 2016). An alternative explanation is dust-trapping at the outer edge of a dead zone. In this scenario, strong accumulations of millimeterand centimeter-sized particles are expected at the location of the outer edge of the dead zone, while the gas is only slightly depleted in the inner part of the disk (Pinilla et al. 2016b). Information on the scattered light emission of SR 24S can give important insights into distinguishing between the two possibilities, since in the dead zone scenario the cavity size in scattered light is expected to have the same size as the millimeter cavity, contrary to the planet-disk interaction scenario (Pinilla et al. 2016b). The resolution of previous near-infrared observations was not enough to detect a potential cavity in small grains in SR 24S (Mayama et al. 2010). Additional information about the distribution of the intermediate grain sizes, which can be obtained by millimeter-wave polarization, can also give significant insights into whether or not planet-disk interaction is the most likely cause for the origin of the cavity in SR 24S (Pohl et al. 2016). Models of internal photoevaporation predict a cavity in gas and dust (and low accretion rates) and therefore this scenario can be ruled out as a potential origin for the millimeter cavity in SR 24S (Owen \& Clarke 2012).
Our analysis for the morphology of the dust emission at 0.45 and $1.30 \mathrm{~mm}$ in SR 24S shows that at longer wavelengths the ring-like emission becomes narrower. This is in agreement with models of dust-trapping in a pressure bump, since larger grains that are traced with longer wavelengths accumulate more efficiently at the location of the pressure maximum (or particle trap). Contrary to model predictions, in the observations there is also a shift of the peak of emission. This shift may result from optically thick emission at the two wavelengths; observations at longer (optically thin) wavelengths are required to see if the peak of emission for larger grains shifts.

The models of particle trapping by planets predict that at early times of evolution ( $\sim 1 \mathrm{Myr})$, the ring-like emission is a radially asymmetric ring, where the outer width of the ring is expected to be higher than the inner width of the ring (e.g., Pinilla et al. 2015). As a result of slow grain growth in the outer disk, the intensity profile at early times of evolution is a ring with an outer tail. At longer times ( $\sim 5 \mathrm{Myr})$, the emission from the models is expected to be a symmetric ring. The dust morphology found from the current observations of SR 24S shows that the emission at both wavelengths is well represented by an almost symmetric ring. The models with the radially asymmetric Gaussian give more similar profiles than the perfect symmetric ring (Figure 6). This suggests that if trapping is occurring due to embedded planets, the trapping process has occurred already for long times of evolution, which is in agreement with the age reported by Wilking et al. (2005) of $\sim 4$ Myr for SR 24S.

In addition, the emission inside the millimeter cavity at $0.45 \mathrm{~mm}$ is less depleted than that at $1.3 \mathrm{~mm}$. This could happen because smaller grains may not be completely filtered at the outer edge of the planet induced-gap, which allows small grains to pass through the gap and replenish the inner disk. The SED of SR 24S shows near-infrared (NIR) excess emission (e.g., Andrews et al. 2011), which suggests the presence of an inner disk. The combination of NIR excess and ring-like emission can be reproduced in models of partial filtration at the outer edge of a gap opened by a massive planet, with changes of the dust dynamics near the water snow line. Without variations of the fragmentation velocities of the particles near the snow line, no NIR excess is produced because even in the case of partial filtration, when small grains pass the planet gap, the growth in the inner disk is so efficient that the grains are lost on very short timescales $(\lesssim 0.1 \mathrm{Myr})$. The required conditions to keep a long-lived inner disk and an outer ring are in agreement with the gas emission peaking inside the millimeter cavity that suggests the presence of low-mass planet (s), allowing the inner disk to be continuously replenished by the outer edge, even at very long times of evolution (Pinilla et al. 2016a).

Analysis of the current data suggests that the morphology of SR 24S disk is not completely azimuthally symmetric and complex asymmetric structures may exist. These asymmetric structures can also be linked with the presence of planets, MRI effects, or from the interaction with the binary system SR 24N. Higher angular resolution observations are needed to confirm the existence of such structures and to better understand their nature and origin.

The current observations of SR $24 \mathrm{~N}$ suggest that this disk is poor on millimeter grains and cold molecular gas. A possible explanation is that the growth of sub-micron-sized particles to larger objects is inhibited by the high dust relative velocities of 
collisions expected around binary systems (Zsom et al. 2011). CIAO observations at the $H$-band showed that the disks in scattered light seem to be extended enough to fill the effective Roche radius of the system, probing tidal interactions between the two disks (Mayama et al. 2010). For the gas, it is possible that only warm gas inside the truncation radii is present (Brown et al. 2013). The truncation radii for close binaries is expected to be $\lesssim 0.4 a$ (specific values depend on the eccentricity and inclination, e.g., Artymowicz \& Lubow 1994; Miranda \& Lai 2015), $a$ being the separation of the two stars. In this case, $a \sim 0$." 2 , which means that the truncation radii is around 0 ". 08 ( $\sim 10 \mathrm{au}$ ), which is smaller than the current resolution of the ALMA observations. An alternative possibility for the lack of millimeter dust and gas in SR $24 \mathrm{~N}$ is that planets already formed in this binary system, depleting the disk in gas and large grains.

\section{Conclusions}

We present ALMA observations of the transition disk SR 24S at 0.45 and $1.3 \mathrm{~mm}$, respectively. Our findings are as follows.

1. The visibilities and images of the continuum emission at the two wavelengths are well described by ring-like emission. The width of such rings is narrower at longer wavelengths, in agreement with particle trapping in pressure bumps. The ring is mostly radially symmetric, suggesting that if the trapping process is due to embedded planets, it must occur for long evolution times.

2. Inside the millimeter cavity, the emission at $0.45 \mathrm{~mm}$ is less reduced than that at $1.3 \mathrm{~mm}(\sim 20 \%$ versus $85 \%$ of depletion with respect to the peak of emission). This could be linked with a constant replenishment of small grains from the outer disk (or partial filtration of particles at the outer edge of a gap). This is agreement with the NIR excess of the SED of SR 24S, i.e., with the presence of an inner disk in the first au.

3. Analysis in the visibility domain reveals a complex morphology of the disk, with a potential asymmetric shape. Higher angular resolution observations are required to confirm such structure(s).

4. We detect ${ }^{13} \mathrm{CO}$ and $\mathrm{C}^{18} \mathrm{O}(J=2-1)$ emission in SR $24 \mathrm{~S}$ disk. The current observations show that the emission of both molecular lines peak at the center of the millimeter cavity, in contrast with most of the transition disks observed so far with ALMA. This suggests that whatever the origin of millimeter cavity is, it allows enough gas to reside in the inner part of the disk, as in the case of dead zones (Pinilla et al. 2016b). Internal photoevaporation is unlikely as a potential origin for the seen structures in SR 24S given the high accretion rate, the large millimeter cavity, and the gas emission peaking in the inner disk

5. There is no detection of the dust-continuum, ${ }^{13} \mathrm{CO}$, or $\mathrm{C}^{18} \mathrm{O}$ emission at the location of the northern component (SR 24N) of this hierarchical triple system SR 24. This suggests that in SR 24N, either planets already formed in this binary system, depleting this circumbinary disk in gas and millimeter grains, or dust growth to millimeter sizes is inhibited in this disk, and only warm gas inside the truncation radii of the binary is present.

6. Assuming physical temperatures from previous modeling for the SR 24S disk, we conclude that the emission at both wavelengths is optically thick close to the location of the ring-like emission. High angular resolution observations at longer wavelengths are needed to investigate potential spatial changes (radial and/or azimuthal) of the dust density distribution through spatially resolved spectral index variations.

The authors thanks the ALMA contact and data reducer, Luke Maud, for helping with the data reduction and calibration. We also acknowledge Catherine Walsh, Marco Tazzari, and Sierk van Terwisga for useful discussions. P.P. acknowledges support by NASA through Hubble Fellowship grant HST-HF251380.001-A awarded by the Space Telescope Science Institute, which is operated by the Association of Universities for Research in Astronomy, Inc., for NASA, under contract NAS 5-26555. Astrochemistry in Leiden is supported by the European Union A-ERC grant 291141 CHEMPLAN, by the Netherlands Research School for Astronomy (NOVA), and by a Royal Netherlands Academy of Arts and Sciences (KNAW) professor prize. T.B. acknowledges funding from the European Research Council (ERC) under the European Union's Horizon 2020 research and innovation program under grant agreement No. 714769. A.N. acknowledges funding from the Science Foundation of Ireland (Grant 13/ERC/I2907). This paper makes use of the following ALMA data: ADS/JAO. ALMA\#2013.1.00091.S and \#2011.0.00724.S. ALMA is a partnership of ESO (representing its member states), NSF (USA) and NINS (Japan), together with NRC (Canada), NSC and ASIAA (Taiwan), and KASI (Republic of Korea), in cooperation with the Republic of Chile. The Joint ALMA Observatory is operated by ESO, AUI/NRAO and NAOJ.

\section{References}

Alexander, R., Pascucci, I., Andrews, S., Armitage, P., \& Cieza, L. 2014, in Protostars and Planets VI, ed. H. Beuther et al. (Tucson, AZ: Univ. Arizona Press), 475

ALMA Partnership, Brogan, C. L., Pérez, L. M., et al. 2015, ApJL, 808, L3 Andrews, S. M., \& Williams, J. P. 2005, ApJL, 619, L175

Andrews, S. M., Wilner, D. J., Espaillat, C., et al. 2011, ApJ, 732, 42

Andrews, S. M., Wilner, D. J., Hughes, A. M., Qi, C., \& Dullemond, C. P. 2010, ApJ, 723, 1241

Andrews, S. M., Wilner, D. J., Zhu, Z., et al. 2016, ApJL, 820, L40

Artymowicz, P., \& Lubow, S. H. 1994, ApJ, 421, 651

Banzatti, A., Testi, L., Isella, A., et al. 2011, A\&A, 525, A12

Birnstiel, T., Klahr, H., \& Ercolano, B. 2012, A\&A, 539, A148

Birnstiel, T., Ricci, L., Trotta, F., et al. 2010, A\&A, 516, L14

Bontemps, S., André, P., Kaas, A. A., et al. 2001, A\&A, 372, 173

Brown, J. M., Pontoppidan, K. M., van Dishoeck, E. F., et al. 2013, ApJ, 770, 94

Bruderer, S., van der Marel, N., van Dishoeck, E. F., \& van Kempen, T. A. 2014, A\&A, 562, A26

Canovas, H., Caceres, C., Schreiber, M. R., et al. 2016, MNRAS, 458, L29 Casassus, S., Wright, C. M., Marino, S., et al. 2015, ApJ, 812, 126

Correia, S., Zinnecker, H., Ratzka, T., \& Sterzik, M. F. 2006, A\&A, 459, 909 Cutri, R. M., Skrutskie, M. F., van Dyk, S., et al. 2003, yCat, 2246

de Boer, J., Salter, G., Benisty, M., et al. 2016, A\&A, 595, A114

de Juan Ovelar, M., Pinilla, P., Min, M., Dominik, C., \& Birnstiel, T. 2016, MNRAS, 459, L85

Dipierro, G., Laibe, G., Price, D. J., \& Lodato, G. 2016, MNRAS, 459, L1 Fedele, D., Carney, M., Hogerheijde, M. R., et al. 2017, A\&A, 600, A72 Flock, M., Ruge, J. P., Dzyurkevich, N., et al. 2015, A\&A, 574, A68 Foreman-Mackey, D., Hogg, D. W., Lang, D., \& Goodman, J. 2013, PASP, 125,306

Ghez, A. M., Neugebauer, G., \& Matthews, K. 1993, AJ, 106, 2005

Ginski, C., Stolker, T., Pinilla, P., et al. 2016, A\&A, 595, A112

Greene, T. P., Wilking, B. A., Andre, P., Young, E. T., \& Lada, C. J. 1994, ApJ, 434, 614

Hildebrand, R. H. 1983, QJRAS, 24, 267 
Loinard, L., Torres, R. M., Mioduszewski, A. J., \& Rodríguez, L. F. 2008, ApJL, 675, L29

Mamajek, E. E. 2008, AN, 329, 10

Mayama, S., Tamura, M., Hanawa, T., et al. 2010, Sci, 327, 306

Miotello, A., van Dishoeck, E. F., Kama, M., \& Bruderer, S. 2016, A\&A, 594, A85

Miranda, R., \& Lai, D. 2015, MNRAS, 452, 2396

Natta, A., Testi, L., \& Randich, S. 2006, A\&A, 452, 245

Nuernberger, D., Brandner, W., Yorke, H. W., \& Zinnecker, H. 1998, A\&A, 330,549

Owen, J. E., \& Clarke, C. J. 2012, MNRAS, 426, L96

Pérez, L. M., Carpenter, J. M., Andrews, S. M., et al. 2016, Sci, 353, 1519

Pérez, L. M., Carpenter, J. M., Chandler, C. J., et al. 2012, ApJL, 760, L17

Pérez, L. M., Chandler, C. J., Isella, A., et al. 2015, ApJ, 813, 41

Pérez, L. M., Isella, A., Carpenter, J. M., \& Chandler, C. J. 2014, ApJL, 783, L13

Pérez, S., Casassus, S., Ménard, F., et al. 2015, ApJ, 798, 85

Pinilla, P., Benisty, M., \& Birnstiel, T. 2012b, A\&A, 545, A81

Pinilla, P., Benisty, M., Birnstiel, T., et al. 2014, A\&A, 564, A51

Pinilla, P., Flock, M., Ovelar, M. d. J., \& Birnstiel, T. 2016b, A\&A, 596, A81

Pinilla, P., Klarmann, L., Birnstiel, T., et al. 2016a, A\&A, 585, A35

Pinilla, P., van der Marel, N., Pérez, L. M., et al. 2015, A\&A, 584, A16

Pohl, A., Kataoka, A., Pinilla, P., et al. 2016, A\&A, 593, A12

Regály, Z., Juhász, A., Sándor, Z., \& Dullemond, C. P. 2012, MNRAS, 419, 1701

Reipurth, B., \& Zinnecker, H. 1993, A\&A, 278, 81

Ricci, L., Testi, L., Natta, A., \& Brooks, K. J. 2010, A\&A, 521, A66

Rosotti, G. P., Ercolano, B., Owen, J. E., \& Armitage, P. J. 2013, MNRAS, 430, 1392

Rosotti, G. P., Juhasz, A., Booth, R. A., \& Clarke, C. J. 2016, MNRAS, 459, 2790

Simon, M., Ghez, A. M., Leinert, C., et al. 1995, ApJ, 443, 625
Sokal, A. D. 1994, arXiv:hep-lat/9405016

Stanke, T., \& Zinnecker, H. 2000, in IAU Symp. 200, Birth and Evolution of Binary Stars, ed. B. Reipurth \& H. Zinnecker (Cambridge: Cambridge Univ. Press), 38

Stolker, T., Dominik, C., Avenhaus, H., et al. 2016, A\&A, 595, A113

Strom, K. M., Strom, S. E., Edwards, S., Cabrit, S., \& Skrutskie, M. F. 1989, AJ, 97, 1451

Tazzari, M., Testi, L., Ercolano, B., et al. 2016, A\&A, 588, A53

Testi, L., Birnstiel, T., Ricci, L., et al. 2014, in Protostars and Planets VI, ed. H. Beuther et al. (Tucson, AZ: Univ. Arizona Press), 339

Trotta, F., Testi, L., Natta, A., Isella, A., \& Ricci, L. 2013, A\&A, 558, A64

van der Marel, N., Pinilla, P., Tobin, J., et al. 2015, ApJL, 810, L7

van der Marel, N., van Dishoeck, E. F., Bruderer, S., et al. 2013, Sci, 340, 1199

van der Marel, N., van Dishoeck, E. F., Bruderer, S., et al. 2016, A\&A, 585, A58

van der Marel, N., van Dishoeck, E. F., Bruderer, S., Pérez, L., \& Isella, A. 2015, A\&A, 579, A106

Walsh, C., Juhász, A., Meeus, G., et al. 2016, ApJ, 831, 200

Whipple, F. L. 1972, in Proc. 21st Nobel Symp., From Plasma to Planet, ed. A. Evlius (New York: Wiley), 211

White, J. A., Boley, A. C., Dent, W. R. F., Ford, E. B., \& Corder, S. 2016, MNRAS, submitted (arXiv:1612.01648)

Wilking, B. A., Lada, C. J., \& Young, E. T. 1989, ApJ, 340, 823

Wilking, B. A., Meyer, M. R., Robinson, J. G., \& Greene, T. P. 2005, AJ, 130,1733

Williams, J. P., \& Best, W. M. J. 2014, ApJ, 788, 59

Zhang, K., Bergin, E. A., Blake, G. A., et al. 2016, ApJL, 818, L16

Zhu, Z., Nelson, R. P., Dong, R., Espaillat, C., \& Hartmann, L. 2012, ApJ, 755,6

Zsom, A., Sándor, Z., \& Dullemond, C. P. 2011, A\&A, 527, A10 